\title{
Nimotuzumab, a novel monoclonal antibody to the epidermal growth factor receptor, in the treatment of non-small cell lung cancer
}

This article was published in the following Dove Press journal:

Lung Cancer: Targets and Therapy

13 October 201 |

Number of times this article has been viewed

\author{
Masayuki Takeda' \\ Isamu Okamoto' \\ Yasumasa Nishimura ${ }^{2}$ \\ Kazuhiko Nakagawa' \\ 'Department of Medical Oncology, \\ ${ }^{2}$ Department of Radiation Oncology, \\ Kinki University Faculty of Medicine, \\ Osaka, Japan
}

\begin{abstract}
The epidermal growth factor receptor (EGFR) is a promising therapeutic target in non-small cell lung cancer, and several therapeutic agents that target this receptor, including EGFR tyrosine kinase inhibitors and monoclonal antibodies to EGFR, have been developed. Such monoclonal antibodies have shown efficacy in combination with chemotherapy and radiotherapy. Nimotuzumab (h-R3) is a humanized monoclonal antibody to EGFR, and its effects in combination with radiation have been sufficiently promising to warrant further investigation in several types of cancer. Furthermore, the typical severe dermatologic toxicities associated with other monoclonal antibodies to EGFR have not been observed with nimotuzumab. We here summarize the results of preclinical studies as well as of previous and ongoing clinical trials of nimotuzumab for the treatment of non-small cell lung cancer.
\end{abstract}

Keywords: non-small cell lung cancer, epidermal growth factor receptor, antibody

\section{Introduction}

Lung cancer is the most common cause of cancer-related death worldwide, with non-small cell lung cancer (NSCLC) accounting for $~ 75 \%$ of all lung cancer cases. ${ }^{1}$ Platinum-based chemotherapy regimens are the standard first-line treatment for individuals with advanced NSCLC, but the efficacy of such regimens has reached a plateau. ${ }^{2}$ New strategies based on a better understanding of tumor biology are thus needed to improve the efficacy of treatment. The epidermal growth factor receptor (EGFR) is a receptor tyrosine kinase that is abnormally upregulated or activated in a variety of tumors. ${ }^{3}$ Deregulation of receptor tyrosine kinases as a result of overexpression or activating mutations is frequently associated with human cancers and results in the promotion of cell proliferation or migration, inhibition of cell death, or the induction of angiogenesis. ${ }^{4}$ The EGFR has thus been identified as an important target in cancer therapy. ${ }^{5}$ Two different types of agent for EGFR inhibition are currently in use or under development for NSCLC: tyrosine kinase inhibitors (TKIs) and monoclonal antibodies (mAbs).

Among EGFR-TKIs, gefitinib and erlotinib have been extensively evaluated in NSCLC, and sensitivity to these drugs has been associated with the presence of somatic mutations in the tyrosine kinase domain of EGFR or with amplification of the EGFR gene. ${ }^{6-8}$ However, these agents have yet to show effective synergy with chemotherapy. Various mAbs to EGFR are also undergoing preclinical studies and clinical trials of their efficacy as anticancer agents. Cetuximab is a chimeric monoclonal antibody that inhibits EGFR by binding to the extracellular domain of the receptor. The relatively modest anticancer activity of cetuximab administered as
Correspondence: Isamu Okamoto

Department of Medical Oncology, Kinki

University Faculty of Medicine,

377-2 Ohno-higashi, Osaka-Sayama,

Osaka 589-85II, Japan

Tel +81723660221

Fax +81723605000

Email chi-okamoto@dotd.med.kindai.ac.jp 
monotherapy in NSCLC patients has prompted evaluation of its use in novel combination regimens. ${ }^{9}$ The safety of cetuximab in combination with the platinum-based two-drug regimens in common use for the treatment of NSCLC has been established in several Phase II studies. ${ }^{10-15}$ Recently, the addition of cetuximab to the regimen of cisplatin and vinorelbine was found to result in improved overall survival in patients with advanced NSCLC positive for EGFR expression. ${ }^{16}$ Subsequent biomarker analysis has shown that patients with high EGFR expression fared better on cetuximab plus chemotherapy than on chemotherapy alone, regardless of histology. The median overall survival in this high-expression group treated with cetuximab plus chemotherapy was 12.0 months, compared with 9.6 months for patients treated with chemotherapy alone (hazard ratio [HR]: $0.73 ; P=0.011)$. In contrast, the patients who had low EGFR expression showed no benefit from the addition of cetuximab to chemotherapy. ${ }^{17}$ The combination of cetuximab with radiotherapy in patients with locally advanced NSCLC has also shown promising results in a Phase II study ${ }^{18}$ and is now being evaluated in a confirmatory trial (Clinicaltrials. gov: NCT00533949).

Nimotuzumab (h-R3) is a humanized mouse mAb to EGFR that binds to the extracellular domain of the receptor and thereby inhibits EGF binding. ${ }^{19}$ In preclinical studies, nimotuzumab has shown antitumor effects in various types of cancer and has been found to enhance the antitumor efficacy of radiation in EGFR-expressing NSCLC cell lines. ${ }^{20,21}$ Nimotuzumab has been approved in more than 20 countries for various indications including pediatric and adult glioma as well as head and neck cancer, nasopharyngeal carcinoma, and esophageal cancer, and it is in clinical trials for various tumor types including NSCLC as well as colorectal, pancreatic, cervical, and breast cancer. This review summarizes the results of preclinical and clinical studies of nimotuzumab and also describes ongoing clinical trials of this drug in NSCLC patients. We also propose future directions for clinical development in this setting.

\section{Mechanism of action}

Nimotuzumab was originally isolated as a mouse immunoglobulin (Ig) G2a antibody, designated R3, which was developed in response to human placental EGFR at the Molecular Immunology Center in Havana, Cuba. The $\mathrm{R} 3 \mathrm{mAb}$ was humanized to reduce its immunogenicity and slow its rate of clearance from the body in humans by grafting its complementarity determining regions onto a human IgG1 molecule, thereby generating h-R3-nimotuzumab. ${ }^{19}$
Whereas it shows similar preclinical and clinical activity when compared with other mAbs to EGFR for certain indications, nimotuzumab has one major advantage: it does not show severe skin toxicity or induce severe hypomagnesemia or gastrointestinal adverse events. ${ }^{22,23}$ The lack of such side effects has been hypothesized to be due to differences in binding affinity for EGFR between nimotuzumab and other mAbs. The dissociation constant $\left(K_{d}\right)$ for the binding of nimotuzumab to EGFR is thus $>10$ times that of other $\mathrm{mAbs}$ to EGFR including cetuximab and panitumumab (Table 1). ${ }^{24-26}$ The binding properties of nimotuzumab and cetuximab were studied in vitro on chip surfaces with cells expressing EGFR at various levels. Whereas cetuximab bound in a monovalent manner to cells with low EGFR expression levels, nimotuzumab required bivalent binding for stable attachment to the cell surface, with the result that it bound selectively to cells with high levels of EGFR expression (Figure 1). ${ }^{27}$ Both antibodies bound bivalently to and accumulated to similar extents on cells with high EGFR expression levels. In situations where EGFR density is low, such as in skin, cetuximab would thus be expected to be more active than nimotuzumab. Its reliance on bivalent binding for effective EGFR blockade allows nimotuzumab to bind robustly to tumor cells overexpressing EGFR while sparing normal cells with a low level of EGFR expression and thereby avoiding unwanted toxicities.

Another possible explanation for the reduced side effects of nimotuzumab has been suggested from the crystal structure of the Fab fragment of nimotuzumab bound to the extracellular region of EGFR. ${ }^{26}$ Nimotuzumab recognizes domain III of the extracellular region of EGFR, and its target site overlaps with both the surface patch recognized by cetuximab and the binding site for epidermal growth factor (EGF). The epitope recognized by nimotuzumab is displaced toward the carboxyl-terminus of EGFR domain III

Table I Comparison of the anti-EGFR mAbs cetuximab and nimotuzumab

\begin{tabular}{|c|c|c|}
\hline & $\begin{array}{l}\text { Cetuximab } \\
\text { (C225) }\end{array}$ & $\begin{array}{l}\text { Nimotuzumab } \\
\text { (h-R3) }\end{array}$ \\
\hline Type of molecule & Chimeric $\mathrm{mAb}$ & Humanized $\mathrm{mAb}$ \\
\hline$K_{d}(\mathrm{M})$ & $1.8 \times 10^{-9}$ & $2.1 \times 10^{-8}$ \\
\hline Ig subclass & $\lg G \mid$ & $\lg G \mid$ \\
\hline \multicolumn{3}{|l|}{ Clinical trial status } \\
\hline In locally advanced NSCLC & $\begin{array}{l}\text { Phase III } \\
\text { (ongoing) }\end{array}$ & $\begin{array}{l}\text { Phase II } \\
\text { (completed) }\end{array}$ \\
\hline In advanced NSCLC & $\begin{array}{l}\text { Phase III } \\
\text { (completed) }\end{array}$ & $\begin{array}{l}\text { Phase I } \\
\text { (ongoing) }\end{array}$ \\
\hline
\end{tabular}

Abbreviations: EGFR, epidermal growth factor receptor; mAb, monoclonal antibody; NSCLC, non-small cell lung cancer. 


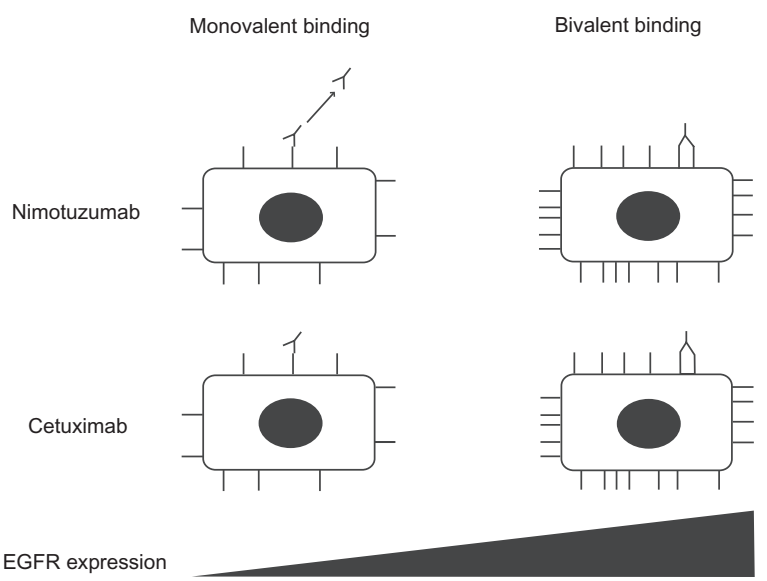

Figure I Schematic representation of the binding properties of nimotuzumab and cetuximab as a function of EGFR density. For cells with a low EGFR density, such as skin cells, both mAbs rely on one arm to bind to the EGFR epitope (monovalent binding) and to exert their effects. The level of EGFR blockade achieved is determined by the specific binding affinity of each $\mathrm{mAb}$, which is lower for nimotuzumab than for cetuximab. For cells with a high EGFR density, such as certain tumor cells, the $\mathrm{mAbs}$ bind via both arms (bivalent binding). Bivalent binding results in the formation of a more stable interaction given that it is dictated by the avidity of binding rather than binding affinity.

Abbreviations: EGFR, epidermal growth factor receptor; mAbs, monoclona antibodies.

compared with the cetuximab binding site. Nimotuzumab sterically interferes with EGF binding, while permitting EGFR domain I to approach domain III and adopt the active conformation. In contrast, cetuximab blocks the binding of EGF and clashes with domain I, preventing the receptor from adopting its active conformation. By interfering only with ligand-dependent EGFR activation, nimotuzumab reduces EGFR signaling to the basal, ligand-independent level, which is required for the survival of normal epithelial cells. Further studies are warranted to determine whether the low toxicity profile of nimotuzumab is indeed explained by the precise structural nature of the interaction between nimotuzumab and the extracellular region of EGFR.

\section{Preclinical data}

The growth-inhibitory potential of nimotuzumab has been tested in vitro and in vivo. Nimotuzumab was found to exhibit a maximum antiproliferative activity of $40 \%$ in A431 cells (a human vulvar epidermoid cell line with a high level of EGFR expression) in vitro. ${ }^{20}$ Analysis of the cell cycle profile of nimotuzumab-treated A431 cells revealed arrest in G1 phase accompanied by a decrease in the proportion of cells in $\mathrm{S}$ phase. This observation, together with the absence of an apparent hypodiploid peak (reflecting apoptotic cells), suggests that nimotuzumab acts principally as a cytostatic rather than cytotoxic agent in vitro. Furthermore, the growth of established subcutaneous A431 tumors in severe combined immunodeficient (SCID) mice was markedly reduced by nimotuzumab as a result of inhibition of both tumor cell proliferation and tumor angiogenesis. ${ }^{20}$

We examined the effects of nimotuzumab in combination with ionizing radiation on human NSCLC cell lines of differing EGFR status. ${ }^{21}$ Nimotuzumab inhibited the EGFinduced or constitutive phosphorylation of EGFR in H292 and Ma-1 cells (Figure 2A), both of which manifest high levels of surface EGFR expression, consistent with the mode of action of this antibody. In contrast, nimotuzumab did not block EGF-induced or constitutive EGFR phosphorylation in H460 and H1975 cells (Figure 2B), both of which exhibit a low level of surface EGFR expression. Given that H292 and $\mathrm{H} 460$ cells both harbor wild-type EGFR alleles whereas Ma-1 and H1975 cells possess mutant EGFR alleles, these observations suggest that the inhibitory effect of nimotuzumab on EGFR signaling depends on the level of EGFR expression at the cell surface, regardless of EGFR mutation status. Consistent with our in vitro results, we found that nimotuzumab enhanced the antitumor effect of radiation on tumors formed by H292 or Ma-1 cells (both with high levels of surface EGFR expression) in nude mice (Figure 2C). Such enhancement was not apparent for tumors formed by $\mathrm{H} 460$ cells (low level of surface EGFR expression) (Figure 2C). These results thus suggest that EGFR mutation is not the major determining factor for enhancement of the antitumor effect of radiation by nimotuzumab, and that such enhancement depends on the tumor expression level of EGFR.

The growth-inhibitory effects of nimotuzumab alone and in combination with radiation on xenografts formed by EGFR-expressing U87MG (human glioblastoma multiforme) cells in NMRI nude mice were also investigated. ${ }^{28}$ Consistent with our results, coadministration of nimotuzumab increased the radiosensitivity of subcutaneous U87MG tumors, resulting in a significant delay in tumor growth. Furthermore, the addition of nimotuzumab to radiation reduced the size of tumors formed by U87MG cells in the brain to a greater extent than did radiation alone, and it inhibited by $40 \%-80 \%$ the increased tumor cell invasion provoked by radiotherapy as well as promoted tumor cell apoptosis. Whereas nimotuzumab also reduced the size of tumor blood vessels and the number of proliferating cells in subcutaneous tumors, cetuximab had no significant antiangiogenic or antiproliferative effects in this model. These results demonstrated the superiority of combined treatment with nimotuzumab and radiation vs radiation alone for glioblastoma multiforme. 
A EGFR high expression

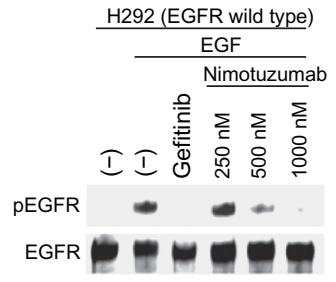

B

\section{EGFR low expression}

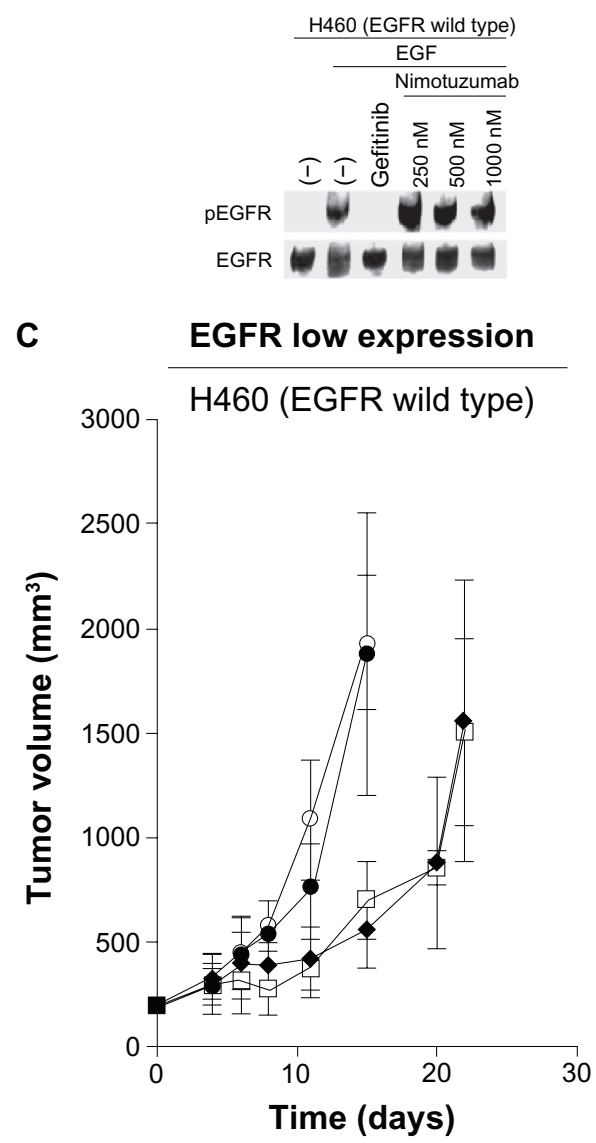

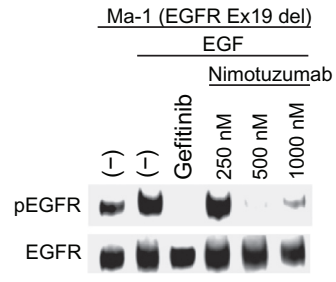

H1975 (EGFR L858R + T790 M)

EGF Nimotuzumab

을 $\sum \sum_{\sum}$

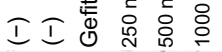

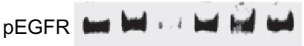

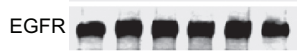

EGFR high expression

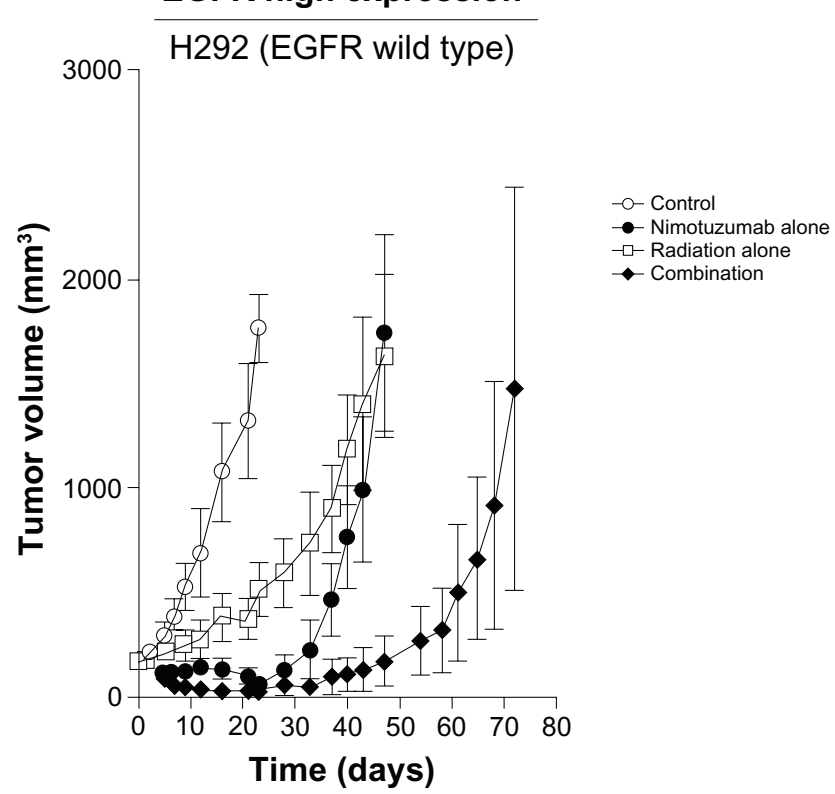

Figure 2 Effects of nimotuzumab on EGFR phosphorylation in NSCLC cells in vitro and on the response of NSCLC cells to radiation in vivo. (A and B) Effect of nimotuzumab on EGFR phosphorylation in NSCLC cells. H292 and Ma-I cells (both of which express EGFR at a high level) (A) as well as H460 and HI 975 cells (both of which express EGFR at a low level) (B) were deprived of serum overnight and then incubated first for 15 minutes in the absence or presence of gefitinib (I0 $\mu M$ ) or the indicated concentrations of nimotuzumab and then for an additional 15 minutes in the additional absence or presence of EGF (I00 ng/mL). Cell lysates were then subjected to immunoblot analysis with antibodies to the Tyr ${ }^{1068}$-phosphorylated form of EGFR (pEGFR) or to total EGFR. H292 and H460 cells possess wild-type EGFR alleles, whereas Ma-I cells harbor a deletion in exon 19 (ExI9 del) of EGFR and HI975 cells harbor both L858R and T790M mutations in EGFR. (C) Effect of nimotuzumab on the response of NSCLC cells to radiation. $\mathrm{H} 460$ and $\mathrm{H} 292$ cells were injected subcutaneously into athymic nude mice. Treatment was initiated when tumors in each group achieved an average volume of $\sim 170-200 \mathrm{~mm}^{3}$. Mice were treated with a single dose of nimotuzumab ( $1.0 \mathrm{mg}$ per mouse) intraperitoneally, a single dose of $\gamma$-radiation (I0 Gy), or neither (control) or both modalities, and tumor volume was determined at the indicated times thereafter.

Note: Data are means \pm SD for seven or eight mice per group. Reprinted from Immunotechnology, Vol 3, Issue I, Mateo et al. Humanization of a mouse monoclonal antibody that blocks the epidermal growth factor receptor: recovery of antagonistic activity. PP 7I-8I.

Copyright (1997), with permission from Elsevier.

Abbreviations: EGFR, epidermal growth factor receptor; EGF, epidermal growth factor; NSCLC, non-small cell lung cancer.

\section{Phase I studies of nimotuzumab}

\section{as a single agent for treatment}

\section{of solid tumors}

The first Phase I trial for nimotuzumab enrolled twelve Cuban patients with advanced epithelial tumors (four ovarian adenocarcinomas as well as four breast, two lung, one stomach, and one renal carcinoma) and administered a one-time intravenous dose of $50,100,200$, or $400 \mathrm{mg}$ of nimotuzumab. ${ }^{29}$ No serious adverse events were observed. Seven of the twelve participants developed mild or moderate 
adverse reactions, consisting mostly of tremors, fever, vomiting, nausea, dryness of mouth, asthenia, hypertension, and flushing. No acneiform rash or other dermatologic toxicity was detected. The area under the curve (AUC) and elimination half-life for the drug increased linearly with dose. Whereas plasma clearance of nimotuzumab decreased as the dose increased from 50 to $200 \mathrm{mg}$, it was similar at doses of 200 and $400 \mathrm{mg}$.

Another Phase I trial was performed to assess the pharmacodynamic effects of escalating weekly intravenous doses of nimotuzumab ranging from 100 to $800 \mathrm{mg}$ administered alone in Caucasian patients with advanced solid cancer. ${ }^{30}$ Tumor and skin biopsy specimens were collected before and 3 weeks after the onset of treatment for immunohistochemical assessment of the expression of EGFR and its downstream signaling molecules. Seventeen patients were enrolled, including one who was not treated with nimotuzumab; most of the patients had colon $(n=11)$ or rectal $(n=2)$ cancer. Although one dose-limiting-toxicity (DLT) was observed at the dose of $100 \mathrm{mg}$ (fatigue of grade 3 ), dose escalation to $800 \mathrm{mg}$ was not associated with any other DLT. No toxicity of grade 4 was observed, and only three patients developed an acneiform rash of grade 1 (18.7\%). Among the 16 patients treated with nimotuzumab, one achieved a partial response (PR, 6.2\%) and eight had stable disease (SD, 50.0\%). The median time to progression (TTP) was 2.4 months. No substantial changes in EGFR, AKT, ERK, or Ki67 immunostaining were observed between the tumor or skin biopsy specimens obtained before treatment onset and those obtained after. Nimotuzumab has thus shown a favorable safety profile when administered as a single agent at doses up to $800 \mathrm{mg}$ in patients with solid tumors.

A Phase I trial was also performed to evaluate the safety profile, maximum tolerated dose (MTD), and DLT for nimotuzumab in patients with advanced solid tumors in Japan. ${ }^{31}$ Nimotuzumab was given intravenously at weekly doses of 100,200 , or $400 \mathrm{mg}$, with four patients enrolled at each dose level. The major adverse event was skin rash of grade 1 or $2(58 \%, 7 / 12)$, and no toxicities of grade 3 or 4 or DLTs were observed. The MTD was thus not determined. Of the 12 patients evaluable for response, SD and progressive disease (PD) were observed in eight (67\%) and four (33\%) patients, respectively, with no objective responses being achieved. Median TTP was 4 months. Nimotuzumab was thus well tolerated at doses up to $400 \mathrm{mg}$ weekly in Japanese patients with advanced solid tumors.

Collectively, these studies demonstrated that nimotuzumab can be administered at doses up to $800 \mathrm{mg}$ per week with moderate toxicity. Importantly, characteristic adverse events seen with other EGFR-targeting antibodies, such as skin toxicities and hypomagnesemia, were not observed with nimotuzumab.

\section{Nimotuzumab in combination with radiotherapy for NSCLC Palliative radiotherapy for advanced NSCLC}

Preclinical data have suggested that nimotuzumab enhances the antitumor activity of ionizing radiation..$^{21}$ It has also shown activity and been well tolerated when administered together with radiation in several clinical trials. A Phase I clinical trial of weekly nimotuzumab $(100,200$, or $400 \mathrm{mg})$ in combination with external radiotherapy (30 or 36 Gy in 3-Gy fractions) was performed in Canadian patients diagnosed with stage IIB, III, or IV NSCLC unsuitable for curative therapy. ${ }^{32}$ The most common adverse events were fatigue, anorexia, chills, pain, and hypophosphatemia (all of grades 1 or 2 in most patients). No severe skin or allergic toxicity was noted. Of 17 patients evaluable for response within the radiation field, twelve showed a PR and five had SD; thus, none had PD. Median progression-free survival (PFS) was 16 weeks, median overall survival (OS) was 60 weeks, and the 1 -year survival rate was $52 \%$. The study did not identify the MTD for this therapeutic combination of nimotuzumab and radiation.

A similarly designed Korean trial documented almost identical results. ${ }^{33}$ Treatment consisted of nimotuzumab $(100,200$, or $400 \mathrm{mg})$ administered weekly with palliative radiotherapy ( 30 to $36 \mathrm{~Gy}$ at $3 \mathrm{~Gy} / \mathrm{day}$ ). Of 15 patients evaluable for response within the radiation field, seven showed a PR and two had SD. Median TTP was 5.4 months, and median OS was 9.8 months. Although one patient developed a DLT (pneumonia of grade 4) at the nimotuzumab dose of $200 \mathrm{mg}$, the MTD was not reached at doses up to $400 \mathrm{mg}$. These two studies thus showed that combination therapy with nimotuzumab and radiation is well tolerated and feasible. A randomized Phase II study comparing nimotuzumab plus palliative radiation with palliative radiation alone is currently ongoing (Clinicaltrials.gov: NCT00369447).

Brain metastases occur in $30 \%-50 \%$ of patients with NSCLC and are associated with a worse prognosis and poorer quality of life. Historically, whole-brain radiation therapy (WBRT) alone was offered for management of brain metastases. A randomized Phase II trial comparing weekly nimotuzumab (200 mg) plus WBRT (40 Gy in 2-Gy fractions) 
with WBRT alone was performed in patients with advanced NSCLC and brain metastasis. ${ }^{34}$ Disease control rate as a primary endpoint was $91.6 \%$ (eleven patients had SD, one showed PD) for the nimotuzumab-radiation arm, compared with $44.4 \%$ (four patients had SD, five showed PD) for the radiation group $(P=0.0039)$. A randomized, double-blind, Phase II study comparing WBRT (30 Gy, in 10 fractions) together with either weekly nimotuzumab (200 mg) or placebo in NSCLC patients with brain metastasis has a target enrollment of 80 patients and is being conducted internationally (Clinicaltrials.gov: NCT00872482). The primary efficacy endpoint is the difference in intracranial disease progression over 6 months.

\section{Curative chemoradiation for locally advanced NSCLC}

Inoperable NSCLC of stage III constitutes $\sim 30 \%$ of all newly diagnosed cases of NSCLC, with the standard of care being combined-modality treatment with both platinum-based chemotherapy and thoracic radiation therapy (TRT). ${ }^{35,36}$ Recent clinical trials have shown that concurrent chemotherapy and radiotherapy (chemoradiation) yields outcomes superior to those of sequential therapy in such patients, with a 3 -year survival rate of $\sim 15 \%-20 \%$ and median OS of 15-20 months, ${ }^{37-39}$ but the efficacy of such regimens has reached a plateau.

Given the preclinical data showing a synergistic effect of nimotuzumab and radiation ${ }^{21}$ and the promising clinical results of nimotuzumab administration in combination with palliative radiotherapy, ${ }^{32,33}$ a Phase II study of nimotuzumab in combination with cisplatin, vinorelbine, and concurrent TRT was performed for stage III NSCLC in Japan (JapicCTI090825). ${ }^{40}$ Treatment consisted of a chemoradiotherapy phase with two cycles of cisplatin $\left(80 \mathrm{mg} / \mathrm{m}^{2}\right)$, vinorelbine (20 mg/m²), and nimotuzumab (200 mg) followed by a consolidation phase with two cycles of these drug regimens (Figure 3). The primary endpoint was tolerability of the drug therapy in combination with concurrent TRT, which was determined from the percentage of patients who completed 60 Gy of radiotherapy within 8 weeks, completed two cycles of chemotherapy, and received more than $75 \%$ of the planned total dose of nimotuzumab. Forty patients, the target sample size, were enrolled. Thirty-four patients (87\%) met the criteria for treatment tolerability, and 38 patients $(97 \%)$ completed 60 Gy of radiotherapy within 8 weeks. Infusion reactions, a skin rash of grade $\geq 3$, radiation pneumonitis of grade $\geq 3$, and nonhematologic toxicities of grade 4 were not observed. For the preliminary efficacy analysis, 37 patients

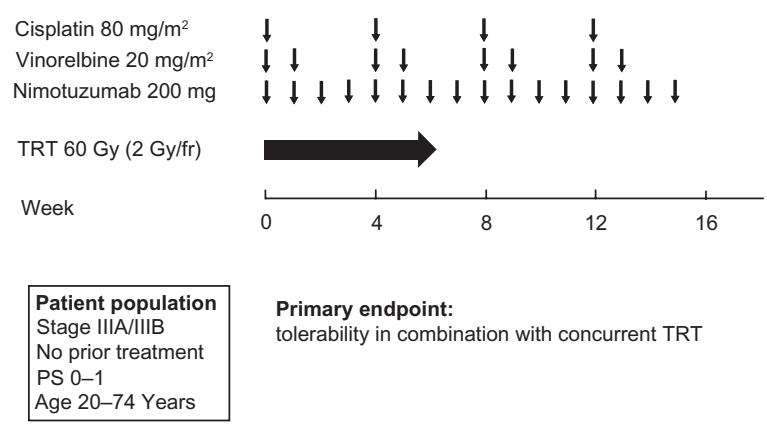

Figure 3 Treatment scheme for the chemoradiotherapy phase and consolidation phase of a Phase II study of nimotuzumab in combination with cisplatin, vinorelbine, and concurrent thoracic radiotherapy (TRT) in patients with stage III NSCLC. ${ }^{40}$ Abbreviations: TRT, thoracic radiotherapy; NSCLC, non-small cell lung cancer; PS, performance status; fr, fraction.

were evaluable with appropriate radiotherapy planning. The response rate was $70 \%$, and the median PFS was 11.1 months. The combination of nimotuzumab with platinum-based chemotherapy and concurrent TRT is thus feasible and shows promising activity for locally advanced NSCLC.

\section{Nimotuzumab in combination with chemotherapy for advanced NSCLC}

Platinum-based doublets with third-generation agents are considered the standard first-line treatment for advanced NSCLC. However, recent advances in cancer cell biology have provided a basis for the development of specific molecularly targeted therapeutic agents, including antibodies to the extracellular domain of EGFR, for the treatment of patients with advanced NSCLC.

The anti-EGFR mAb cetuximab prolonged survival in patients with advanced NSCLC when combined with platinum-based chemotherapy in the FLEX Phase III trial. ${ }^{16}$ A supportive study, BMS099, did not achieve its primary PFS endpoint, although an improvement in overall response rate and a favorable OS trend were observed when cetuximab was added to chemotherapy. ${ }^{41} \mathrm{~A}$ meta-analysis evaluated the effect of adding cetuximab to chemotherapy for first-line treatment of advanced NSCLC. The pooled HR for OS $(0.87$; $P=0.004$ ) was in favor of cetuximab plus chemotherapy, which also gave rise to a higher overall response rate (relative risk [RR]: $1.19 ; P=0.013)$. However, the analysis failed to show the benefit of cetuximab plus chemotherapy for PFS (HR: $0.91 ; P=0.06$ ) or 1-year survival rate (RR: 1.10; $P=0.172){ }^{42}$

A Phase I study of nimotuzumab in combination with cisplatin and docetaxel in chemotherapy-naïve patients with advanced NSCLC has begun recruitment of patients (Clinicaltrials.gov: NCT00985998). Patients will receive 
nimotuzumab at doses of 200,400 , or $600 \mathrm{mg}$ on days 1,8 , and 15 plus cisplatin $\left(75 \mathrm{mg} / \mathrm{m}^{2}\right)$ and docetaxel $\left(75 \mathrm{mg} / \mathrm{m}^{2}\right)$ on day 1 of a 3 -week cycle for up to six cycles. The primary endpoint is safety. In the second-line therapy setting, a randomized Phase II trial evaluating the efficacy of docetaxel with or without nimotuzumab in advanced NSCLC is ongoing (NCT 00983047). Docetaxel is administered at a dose of $75 \mathrm{mg} / \mathrm{m}^{2}$ on day 1 of a 3-week cycle for up to six cycles, whereas nimotuzumab at a dose of $200 \mathrm{mg}$ per week is administered during chemotherapy and is continued after its completion until the detection of disease progression or unacceptable toxicity. The total sample size will be 52 eligible patients in each treatment arm, for a total of 104 eligible patients. The primary endpoint is OS.

\section{Predictive biomarkers Molecular predictors of response}

Many surrogate markers, including molecular markers and physical signs, for the selection of patients who are likely to benefit from molecularly targeted therapy, including treatment with mAbs to EGFR, have been investigated. Whereas the presence of an $E G F R$ mutation is the strongest predictor of sensitivity to EGFR-TKIs, ${ }^{6-8}$ the relation between $E G F R$ mutations and the response to anti-EGFR mAbs remains unclear. A preclinical study examined the effects of gefitinib and cetuximab on NSCLC cell lines with mutant EGFR alleles in vitro but failed to reveal a significant association between $E G F R$ mutation status and specific outcome of cetuximab treatment. ${ }^{43}$ In the FLEX trial, EGFR mutation status was determined in 293 of 1125 patients, with 45 patients $(15.4 \%)$ being found to harbor an EGFR-activating mutation. ${ }^{44}$ The presence of such an EGFR mutation was associated with better survival in patients treated with chemotherapy with or without cetuximab but was not predictive of benefit derived from the addition of cetuximab. EGFR mutations were thus of prognostic value in this patient population. Similar findings were obtained in the BMS099 trial, in which EGFR-activating mutations were found in $17(10 \%)$ of 167 patients but were not predictive of benefit for the addition of cetuximab to chemotherapy. ${ }^{45}$ In a preclinical study, we found that nimotuzumab enhanced the antitumor efficacy of radiation in certain human NSCLC cell lines harboring either wild-type or mutant $E G F R,{ }^{21}$ consistent with the notion that EGFR mutation is not the major determining factor for enhancement of the antitumor effect of radiation by nimotuzumab. Together, these various data indicate that the presence of an EGFR mutation is not a prerequisite for a response to anti-EGFR $\mathrm{mAbs}$.
No clear association has emerged between the level of EGFR expression measured by immunohistochemical analysis and the response to the anti-EGFR mAbs cetuximab or panitumumab in metastatic colorectal cancer. Conflicting results regarding the relation between EGFR expression and outcome in NSCLC patients treated with mAbs to EGFR have been reported. Analysis of EGFR expression levels in tumors of patients participating in the FLEX study showed that, among those with a high level of EGFR expression, the response rate in the cetuximab-plus-chemotherapy arm was $44.4 \%$ compared with $28.1 \%$ in the chemotherapyalone $\operatorname{arm}(P=0.002)$. Among patients with a low level of EGFR expression, there was no difference in response rate between the two treatment arms $(32.6 \%$ for cetuximab plus chemotherapy, vs $29.6 \%$ for chemotherapy alone). ${ }^{17}$ With regard to nimotuzumab, we have shown that enhancement of the growth-inhibitory effect of radiation by nimotuzumab in NSCLC cell lines depends on the expression level of EGFR both in vitro and in a xenograft model. ${ }^{19}$ Analysis of tumor samples from patients with locally advanced head and neck cancer treated with radiotherapy plus nimotuzumab did not detect an association between EGFR expression and treatment outcome, although this finding may have been due to the small number of patients. ${ }^{20}$ EGFR expression was also evaluated immunohistochemically in ten NSCLC specimens in a Phase I trial of nimotuzumab plus palliative radiation therapy, but again there was no statistically significant association between EGFR expression and treatment response or outcome because of the limited sample size. ${ }^{30}$ No definitive conclusions can thus be drawn at this time concerning the predictive utility of immunohistochemically determined EGFR expression level for the treatment of NSCLC patients with anti-EGFR mAbs, and further studies are therefore warranted.

\section{Clinical predictors of response}

Patients treated with EGFR inhibitors frequently develop a rash characterized by inflammatory papules and pustules on the scalp, face, neck, and upper trunk. To evaluate the association between rash and cetuximab efficacy in patients with NSCLC, a subgroup analysis of patients from the FLEX study was performed. Patients in the chemotherapy plus cetuximab group with first-cycle rash had significantly prolonged OS compared with patients in the same treatment group without first-cycle rash (median 15.0 months vs 8.8 months; $P<0.0001) .{ }^{46}$ OS for patients without first-cycle rash was similar to that of patients who received chemotherapy alone (median 8.8 months vs 10.3 months; $P=0.36$ ). Rash may 
therefore be a surrogate marker of target inhibition and activity of cetuximab-based chemotherapies.

In contrast, these side effects are rare in cases of nimotuzumab use and are mainly limited to grade 1 or 2 adverse events. ${ }^{25}$ Most conspicuously, the typical grade 3 or 4 skin toxicity found in other anti-EGFR drugs has thus far remained virtually absent in nimotuzumab trials. ${ }^{22}$ Therefore, in contrast to the other EGFR mAbs, attempts at finding clinical predictive markers specifically for nimotuzumab have to date not been forthcoming.

\section{Conclusions}

There is a need to improve the outcome in NSCLC. Nimotuzumab is an EGFR-targeting mAb that has the potential for use as a sensitizer to radio- or chemotherapy in patients with NSCLC positive for EGFR expression. The key feature of clinical experience with nimotuzumab to date is that it does not induce the skin toxicities of grade 3 or 4 that are common to other anti-EGFR mAbs. If nimotuzumab is shown to be clinically beneficial in ongoing and upcoming Phase II and III trials, its benign side effect profile will make it an attractive therapeutic option compared with other antiEGFR mAbs. Given that previous studies have not identified predictive molecular markers for nimotuzumab treatment, mostly as a result of a small sample size, further studies to establish such markers are also needed.

\section{Disclosure}

The authors declare no conflicts of interest in this work.

\section{References}

1. Hoffman PC, Mauer AM, Vokes EE. Lung cancer. Lancet. 2000; 355(9202):479-485.

2. Schiller JH, Harrington D, Belani CP, et al. Comparison of four chemotherapy regimens for advanced non-small-cell lung cancer. $N$ Engl $J$ Med. 2002;346(2):92-98.

3. Baselga J. Why the epidermal growth factor receptor? The rationale for cancer therapy. Oncologist. 2002;7 Suppl 4:2-8.

4. Gschwind A, Fischer OM, Ullrich A. The discovery of receptor tyrosine kinases: targets for cancer therapy. Nat Rev Cancer. 2004; 4(5):361-370.

5. Baselga J, Arteaga CL. Critical update and emerging trends in epidermal growth factor receptor targeting in cancer. J Clin Oncol. 2005; 23(11):2445-2459.

6. Lynch TJ, Bell DW, Sordella R, et al. Activating mutations in the epidermal growth factor receptor underlying responsiveness of non-small-cell lung cancer to gefitinib. N Engl J Med. 2004;350(21):2129-2139.

7. Paez JG, Janne PA, Lee JC, et al. EGFR mutations in lung cancer: correlation with clinical response to gefitinib therapy. Science. 2004; 304(5676):1497-1500.

8. Pao W, Miller V, Zakowski M, et al. EGF receptor gene mutations are common in lung cancers from "never smokers" and are associated with sensitivity of tumors to gefitinib and erlotinib. Proc Natl Acad Sci USA. 2004;101(36):13306-13311.
9. Hanna N, Lilenbaum R, Ansari R, et al. Phase II trial of cetuximab in patients with previously treated non-small-cell lung cancer. J Clin Oncol. 2006;24(33):5253-5258.

10. Robert F, Blumenschein G, Herbst RS, et al. Phase I/IIa study of cetuximab with gemcitabine plus carboplatin in patients with chemotherapy-naive advanced non-small-cell lung cancer. J Clin Oncol. 2005;23(36):9089-9096.

11. Thienelt CD, Bunn PA Jr, Hanna N, et al. Multicenter phase I/II study of cetuximab with paclitaxel and carboplatin in untreated patients with stage IV non-small-cell lung cancer. J Clin Oncol. 2005;23(34): 8786-8793.

12. Borghaei H, Langer CJ, Millenson M, et al. Phase II study of paclitaxel, carboplatin, and cetuximab as first line treatment, for patients with advanced non-small cell lung cancer (NSCLC): results of OPN-017. J Thorac Oncol. 2008;3(11):1286-1292.

13. Belani CP, Schreeder MT, Steis RG, et al. Cetuximab in combination with carboplatin and docetaxel for patients with metastatic or advancedstage nonsmall cell lung cancer: a multicenter phase 2 study. Cancer. 2008;113(9):2512-2517.

14. Socinski MA, Saleh MN, Trent DF, et al. A randomized, phase II trial of two dose schedules of carboplatin/paclitaxel/cetuximab in stage IIIB/IV non-small-cell lung cancer (NSCLC). Ann Oncol. 2009;20(6):1068-1073.

15. Rosell R, Robinet G, Szczesna A, et al. Randomized phase II study of cetuximab plus cisplatin/vinorelbine compared with cisplatin/ vinorelbine alone as first-line therapy in EGFR-expressing advanced non-small-cell lung cancer. Ann Oncol. 2008;19(2):362-369.

16. Pirker R, Pereira JR, Szczesna A, et al. Cetuximab plus chemotherapy in patients with advanced non-small-cell lung cancer (FLEX): an openlabel randomised phase III trial. Lancet. 2009;373(9674):1525-1531.

17. O'Byrne K, Eberhardt WEE, Störkel S, et al. Use of a continuous EGFR IHC scoring method reveals association between tumor response and EGFR expression levels for patients receiving CT + cetuximab versus CT alone in the phase III trial FLEX in advanced NSCLC. $J$ Thorac Oncol. 2010:5 Suppl 7:S558-S559.

18. Blumenschein GR Jr, Paulus R, Curran WJ, et al. Phase II study of cetuximab in combination with chemoradiation in patients with stage iiia/b non-small-cell lung cancer: RTOG 0324. J Clin Oncol. 2011;29(17):2312-2318.

19. Mateo C, Moreno E, Amour K, Lombardero J, Harris W, Perez R. Humanization of a mouse monoclonal antibody that blocks the epidermal growth factor receptor: recovery of antagonistic activity. Immunotechnology. 1997;3(1):71-81.

20. Crombet-Ramos T, Rak J, Perez R, Viloria-Petit A. Antiproliferative, antiangiogenic and proapoptotic activity of h-R3: A humanized antiEGFR antibody. Int J Cancer. 2002;101(6):567-575.

21. Akashi Y, Okamoto I, Iwasa T, et al. Enhancement of the antitumor activity of ionising radiation by nimotuzumab, a humanised monoclonal antibody to the epidermal growth factor receptor, in non-small cell lung cancer cell lines of differing epidermal growth factor receptor status. Br J Cancer. 2008;98(4):749-755.

22. Crombet T, Osorio M, Cruz T, et al. Use of the humanized anti-epidermal growth factor receptor monoclonal antibody h-R3 in combination with radiotherapy in the treatment of locally advanced head and neck cancer patients. J Clin Oncol. 2004;22(9):1646-1654

23. Rodriguez MO, Rivero TC, del Castillo Bahi R, et al. Nimotuzumab plus radiotherapy for unresectable squamous-cell carcinoma of the head and neck. Cancer Biol Ther. 2010;9(5):343-349.

24. Arteaga ME, Ledon N, Casaco A, et al. Systemic and skin toxicity in Cercopithecus aethiops sabaeus monkeys treated during 26 weeks with a high intravenous dose of the anti-epidermal growth factor receptor monoclonal antibody Nimotuzumab. Cancer Biol Ther. 2007;6(9):1390-1395.

25. Boland WK, Bebb G. Nimotuzumab: a novel anti-EGFR monoclonal antibody that retains anti-EGFR activity while minimizing skin toxicity. Expert Opin Biol Ther. 2009;9(9):1199-1206. 
26. Talavera A, Friemann R, Gomez-Puerta S, et al. Nimotuzumab, an antitumor antibody that targets the epidermal growth factor receptor, blocks ligand binding while permitting the active receptor conformation. Cancer Res. 2009;69(14):5851-5859.

27. Garrido G, Tikhomirov IA, Rabasa A, et al. Bivalent binding by intermediate affinity of nimotuzumab: a contribution to explain antibody clinical profile. Cancer Biol Ther. 2011;11(4):373-382.

28. Diaz Miqueli A, Rolff J, Lemm M, Fichtner I, Perez R, Montero E. Radiosensitisation of U87MG brain tumours by anti-epidermal growth factor receptor monoclonal antibodies. Br J Cancer. 2009;100(6): 950-958.

29. Crombet T, Torres L, Neninger E, et al. Pharmacological evaluation of humanized anti-epidermal growth factor receptor, monoclonal antibody h-R3, in patients with advanced epithelial-derived cancer. J Immunother. 2003;26(2):139-148.

30. You B, Brade A, Magalhaes JM, et al. A dose-escalation phase I trial of nimotuzumab, an antibody against the epidermal growth factor receptor, in patients with advanced solid malignancies. Invest New Drugs. 2011;29(5):996-1003.

31. Boku N, Yamazaki K, Yamamoto N, et al. Phase I study of nimotuzumab, a humanized anti-epidermal growth factor receptor (EGFR) IgG1 monoclonal antibody in patients with solid tumors in Japan [abstract]. J Clin Oncol. 2009;27 Suppl 15s:e14574.

32. Bebb G, Smith C, Rorke S, et al. Phase I clinical trial of the anti-EGFR monoclonal antibody nimotuzumab with concurrent external thoracic radiotherapy in Canadian patients diagnosed with stage IIb, III or IV non-small cell lung cancer unsuitable for radical therapy. Cancer Chemother Pharmacol. 2011;67(4):837-845.

33. Choi HJ, Sohn JH, Lee CG, et al. A phase I study of nimotuzumab in combination with radiotherapy in stages IIB-IV non-small cell lung cancer unsuitable for radical therapy: Korean results. Lung Cancer. 2011;71(1):55-59.

34. Macias A, Neninger E, Santiesteban E, et al. Preliminary results of a phase II clinical trial of the anti EGFR monoclonal antibody Nimotuzumab in combination with whole brain radiation therapy in patients diagnosed with advanced non-small cell lung cancer tumors unresectable brain metastases [abstract]. Proceedings of the 20th EORTC-NCIAACR Annual Meeting; October 21-24, 2008; Geneva, Switzerland. 2008:505.

35. Dillman RO, Seagren SL, Propert KJ, et al. A randomized trial of induction chemotherapy plus high-dose radiation versus radiation alone in stage III non-small-cell lung cancer. $N$ Engl J Med. 1990;323(14):940-945.

36. Sause W, Kolesar P, Taylor SI, et al. Final results of phase III trial in regionally advanced unresectable non-small cell lung cancer: Radiation Therapy Oncology Group, Eastern Cooperative Oncology Group, and Southwest Oncology Group. Chest. 2000;117(2):358-364
37. Furuse K, Fukuoka M, Kawahara M, et al. Phase III study of concurrent versus sequential thoracic radiotherapy in combination with mitomycin, vindesine, and cisplatin in unresectable stage III non-small-cell lung cancer. J Clin Oncol. 1999;17(9):2692-2699.

38. Curran W, Scott C, Langer C, et al. Longterm benefit is observed in a phase III comparison of sequential vs concurrent chemo-radiation for patients with unresected stage III NSCLC: RTOG 9410 [abstract]. Proc Am Soc Clin Oncol. 2003;22:2499.

39. Yamamoto N, Nakagawa K, Nishimura Y, et al. Phase III study comparing second- and third-generation regimens with concurrent thoracic radiotherapy in patients with unresectable stage III non-small-cell lung cancer: West Japan Thoracic Oncology Group WJTOG0105. J Clin Oncol. 2010;28(23):3739-3745.

40. Tanaka K, Okamoto I, Yamamoto N, et al. Phase 2 study of nimotuzumab in combination with concurrent chemoradiotherapy (CRT) in patients with locally advanced non-small cell lung cancer (NSCLC) [abstract]. Proceedings of the European Multidisciplinary Cancer Congress; September 23-27, 2011; Stockholm, Sweden: abstract 9.044.

41. Lynch TJ, Patel T, Dreisbach L, et al. Cetuximab and first-line taxane/ carboplatin chemotherapy in advanced non-small-cell lung cancer: results of the randomized multicenter phase III trial BMS099. J Clin Oncol. 2010;28(6):911-917.

42. Lin H, Jiang J, Liang X, Zhou X, Huang R. Chemotherapy with cetuximab or chemotherapy alone for untreated advanced non-small-cell lung cancer: a systematic review and meta-analysis. Lung Cancer. 2010;70(1):57-62.

43. Mukohara T, Engelman JA, Hanna NH, et al. Differential effects of gefitinib and cetuximab on non-small-cell lung cancers bearing epidermal growth factor receptor mutations. J Natl Cancer Inst 2005;97(16):1185-1194.

44. Gatzemeier U, Paz-Ares L, Rodrigues Pereira J, von Pawel J, Ramlau R, Roh J. Molecular and clinical biomarkers of cetuximab efficacy: data from the phase III FLEX study in non-small cell lung cancer (NSCLC) [abstract]. J Thorac Oncol. 2009;4(Suppl 1):S324.

45. Khambata-Ford S, Harbison CT, Hart LL, et al. Analysis of potential predictive markers of cetuximab benefit in BMS099, a phase III study of cetuximab and first-line taxane/carboplatin in advanced non-smallcell lung cancer. J Clin Oncol. 2010;28(6):918-927.

46. Gatzemeier U, von Pawel J, Vynnychenko I, et al. First-cycle rash and survival in patients with advanced non-small-cell lung cancer receiving cetuximab in combination with first-line chemotherapy: a subgroup analysis of data from the FLEX phase 3 study. Lancet Oncol. 2011; 12(1):30-37.
Lung Cancer: Targets and Therapy

\section{Publish your work in this journal}

Lung Cancer: Targets and Therapy is an international, peer-reviewed, open access journal focusing on lung cancer research, identification of therapeutic targets and the optimal use of preventative and integrated treatment interventions to achieve improved outcomes, enhanced survival and quality of life for the cancer patient. Specific topics covered in the journal include:

\section{Dovepress}

Epidemiology, detection and screening; Cellular research and biomarkers; Identification of biotargets and agents with novel mechanisms of action; Optimal clinical use of existing anticancer agents, including combination therapies; Radiation and surgery; Palliative care; Patient adherence, quality of life, satisfaction; Health economic evaluations. 